\title{
El Instituto Nacional de Seguridad Social: Una organización al servicio de sus clientes
}

\section{Alfredo González-Posada Sánchez*}

\section{Introducción}

El Instituto Nacional de Seguridad Social, órgano de la Administración Pública que administra más de 6,5 millones de nuevas pensiones, gestiona cada año más de medio millón de pensiones y otras prestaciones, y dispone de más de doce mil empleados públicos, con estructura gestora en cada provincia, es, en la actualidad, una organización comprometida con la gestión y atención personalizada de los ciudadanos que demandan sus servicios.

Sin embargo, no siempre fue de este modo, ya que también han existido etapas en las que la consideración del ciudadano como administrado tenía mayor preponderancia con todos los inconvenientes que dicho concepto conlleva.

De ahí que considero conveniente hacer a continuación, en primer lugar, una breve reflexión sobre la organización y, en segundo lugar, una más detenida referencia sobre cuales han sido las causas que justificaron el cambio de orientación, las principales políticas impulsoras del mismo, los resultados principales y la percepción que de ellos han experimentado los ciudadanos que se comunican con el mismo.

\section{Antecedentes}

El Instituto nace, administrativamente hablando, en 1978 por aplicación del Real Decreto-ley 38/1978, de 16 de noviembre, que reordena las Entidades Gestoras de la Seguridad Social. Las variaciones introducidas por la citada norma perseguían, entre otras finalidades, la formación de un organismo que aglutinara las funciones del Instituto Nacional de Previsión y del Mutualismo Laboral en materia de gestión y administración de las pensiones y otras prestaciones económicas del sistema.

De este modo, se integran en una sola organización las competencias, las funciones, las personas y los procedimientos gestores, los cuales presentaban marcadas diferencias gestoras, tenían distinto nivel de desarrollo tecnológico y disponían de elementos culturales propios y de carácter divergente.

Este proceso de integración resultó más complejo al incluir a las Mutualidades Laborales que en algunos supuestos disponían de infraestructura y recursos propios en Madrid y en otras provincias. Por ello, en la evolución que ha tenido el Instituto Nacional de Seguridad Social (en adelante INSS), se podrían distinguir las siguientes etapas:

- La comprendida entre el nacimiento y finales de 1980, que se caracteriza por ser una etapa de ajuste estructural, en la que se suprimen las líneas jerárquicas superpuestas de las instituciones extinguidas, se desarrolla la estructura de gestión unitaria y se inician los trabajos de unificación de procedimientos.

- Entre 1980 y 1984 la organización desarrolla un proceso de clarificación de los contenidos funcionales de las unidades administrativas, delimita el sistema de rela- 
ciones de dependencia entre las direcciones provinciales y las agencias situadas en el ámbito provincial e inicia, de forma parcial, el control de las pensiones ya reconocidas. Característica común a la gestión de esta etapa es la mayor consideración otorgada al cumplimiento del procedimiento y a las garantías del administrado, que a la incorporación de otras medidas más innovadoras en la prestación de los servicios.

- En los tres años siguientes (1984-1987), una de las principales novedades gestoras consistió en la introducción de los primeros mecanismos de control y seguimiento de las pensiones solicitadas y reconocidas. Ello permitió un mejor conocimiento de la realidad gestora provincial, así como la formulación de los primeros indicadores de gestión dotando a la dirección de un elemento de análisis de sus actividades. También la construcción del sistema estadístico posibilitó la determinación de las bolsas de expedientes pendientes de tramitar y resolver, así como la mejor asignación de efectivos y recursos en función de las demandas y solicitudes existentes.

\section{El cambio en el enfoque gestor}

Hasta ese momento los esfuerzos realizados por los responsables de la Institución habían permitido acortar en cierta medida los tiempos de tramitación de las pensiones empleando la fórmula de incorporar importantes recursos que, si bien en un principio, produjo un mayor grado de eficacia, a medida que transcurría el tiempo, hizo que la misma fuera decreciendo. Es precisamente en 1988 cuando se incorpora un nuevo enfoque en la gestión del INSS, pues se enfatiza la importancia de conceptos tales como la oferta de servicios, la atención personalizada y en definitiva se inicia un proceso de transición en el que la percepción del administrado se hace menos en sentido juridicista y más en su condición de ciudadano demandante de servicios que deben satisfacerse de modo más ágil.

El punto de inflexión institucional coincidió con la formulación de un diagnóstico de la situación, previo el oportuno período de reflexión que permitió la identificación de los siguientes puntos débiles.

- La organización no estaba suficientemente consolidada, ya que pervivían de modo inconexo aptitudes, comportamientos y formas de gestionar, herederos de la fusión inicial.
- No se percibía de manera concluyente cuáles eran las áreas clave para el desarrollo de las funciones encomendadas.

- Los programas de trabajo y de actuaciones carecían de un alto grado de definición y priorización lo cual provocaba conflictos entre los responsables en el proceso de ejecución.

- Las políticas gestoras de recursos humanos presentaban una muy deficiente instrumentación lo que dificultaba una adecuada utilización y distribución de los mismos.

- Los diferentes sistemas de información de que disponía la dirección tenían un carácter parcial, fruto del carácter sectorial con que habían sido concebidos y carecían de la oportuna coordinación.

- Las instalaciones físicas presentaban un alto grado de amortización y no se habían previsto y desarrollado políticas inversoras que paliasen las deficiencias existentes.

Esta reflexión también puso de manifiesto la existencia de problemas que, bien por su complejidad o por su concurrencia en el tiempo, demandaban la adopción de decisiones de diversa magnitud. Los principales problemas detectados fueron los siguientes:

- La planificación de los recursos humanos no siempre respondía a las necesidades organizativas y los mecanismos utilizados en la provisión de los puestos de trabajo no siempre tenían en cuenta los méritos y aptitudes precisas para su desempeño.

- Existía una indefinición de los perfiles correspondientes a los puestos de trabajo y se carecía de una formulación de los parámetros definitorios de la carrera de servicios en la organización.

- Los funcionarios de las unidades periféricas presentaban una insuficiente cualificación y especialmente los de las áreas de atención al ciudadano. Tampoco se encontraban explicitadas las funciones del personal de dichas unidades.

- Los planes de formación presentaban grandes lagunas en su conceptualización y diseño, sin que coadyuvasen a la motivación e incentivación del personal.

- El modelo organizativo era excesivamente rígido, burocratizado y carecía de respuesta para las demandas innovadoras en materia de seguridad e higiene.

- Las dotaciones de puestos staff eran insuficientes, y no se disponía de recursos humanos expertos en áreas estratégicas que favoreciesen la toma de decisiones.

- La oferta de servicios era desigual en las agencias del INSS, con todo lo que suponía de trato discriminato- 
rio para los ciudadanos, constatándose un desequilibrio en la demanda de servicios entre unidades, lo que provocaba efectos no deseados tales como malestar entre los usuarios, dilación en la prestación del servicion, etcétera.

- Los soportes informáticos para la gestión de las prestaciones y de los recursos en general eran claramente insuficientes en las unidades periféricas, lo que obligaba a reducir la oferta de servicios.

- La gestión de las pensiones y otras prestaciones presentaba unos tiempos de resolución muy dilatados y los mecanismos de seguimiento y medición carecían de la suficiente idoneidad.

- La imagen institucional resultaba inadecuada, con una personalidad poco definida por los solapamientos con otras instituciones del entorno y anticuada por el efecto que sobre los ciudadanos causaba la red de agencias.

- El tratamiento de los datos de los expedientes gestionados resultaba muy complejo y exigía múltiples esfuerzos, con lo que la retroalimentalización en la cúpula directiva de la gestión realizada en las provincias resultaba inadecuada.

- El volumen de documentación exigido resultaba excesivo, y los documentos remitidos a los solicitantes contenían previsiones técnico-jurídicas de difícil comprensión, lo cual hacía que la percepción del ciudadano respecto de la Administración fuera la de una organización inaccesible, lejana y opaca.

- Tampoco se tenía una consideración omnicomprensiva de la comunicación, sin que se le prestase la suficiente importancia a los vehículos de transmisión de la información.

- No se disponía de sistemas de evaluación de los servicios prestados lo cual impedía conocer el nivel de satisfacción de los ciudadanos.

Para la corrección de las disfuncionalidades detectadas y la superación de la situación se han diseñado y puesto en funcionamiento, en los seis últimos años, un conjunto de políticas y estrategias cuyo denominador común consistía en destacar que lo prioritario en la gestión de las pensiones era el ciudadano al que se prestaba un servicio $y / 0$ se reconocia una pensión.

En este sentido, se diseñó un plan que contenía múltiples programas de trabajo y que se agrupaban en torno a tres áreas: la de Comunicación, la de Organización y la de Gestión de Productos y Servicios.
Las políticas de comunicación se han dirigido a desarrollar y potenciar la atención y la información que se presta a los diferentes colectivos con los que, de forma sistemática y necesaria, entra en contacto la institución. Ello ha hecho necesario el desarrollo de programas de difusión, de información de anticipación y campanas especializadas para distintos medios de comunicación.

Las políticas de organización han consistido, por un lado, en la definición de un nuevo modelo de organización en el que se distingue entre los centros de Atención e Información (en adelante CAISS), que se configuran como centros abiertos, donde se concentra la atención e información a los ciudadanos y los centros de producción o de gestión (Direcciones provinciales) donde se resuelven las prestaciones demandadas y que tiene un carácter de centro cerrado sin atención a los clientes. Por otro lado, estas políticas pretenden superar las diferentes carencias que para la gestión de recursos humanos se indicaron con anterioridad.

Las políticas de gestión tienen como finalidad esencial desarrollar una gran capacidad de gestión basada en la agilidad, la celeridad y la eficacia. Ahora bien, esto supone dotarlas de una gran flexibilidad para poder introducir, con la mayor rapidez y sin producir perturbaciones en la actividad ordinaria, los cambios en el marco jurídico o procedimental que cada día se estimen necesarios.

\section{Las áreas claves para el cambio}

Es evidente que la traducción de estas políticas en programas de trabajo ha producido una dinamización general de la organización, algunos de cuyos resultados se analizan en el siguiente apartado. Sin embargo, creo que fundamentalmente han sido tres las áreas donde el esfuerzo de transformación y modernización han constiruido las palancas sobre las que se ha fundamentado el proceso de cambio institucional.

\section{Los procedimientos}

La incorporación sucesiva de las medidas sobre simplificación documental, la definición de nuevos procedimientos gestores y la introducción de elementos de racionalización en la gestión de las pensiones, han constituido factores muy eficaces y útiles para la gestión de los expedientes de prestaciones económicas, que hoy en día son los principales "productos" del INSS. 
La simplificación documental establecida por Resolución de la Secretaría General para la Seguridad Social de 2 de febrero de 1988 (BOE de 5 de febrero), supuso la reducción en el número de documentos exigibles a los solicitantes de prestaciones. Frente a los 10 documentos exigibles se pasó a una situación en la que sólo se exigen tres con carácter general. De esta manera se logró extraer del circuito de la burocracia más de 700.000 documentos al año que repercutían negativamente en el trámite de los expedientes y alargaba la cadena de gestión al exigir más medios y controles.

Para ello fue preciso conseguir un mejor aprovechamiento de la información interna obrante en los bancos de datos de la Seguridad Social, la coordinación con otras Administraciones, evitando a los ciudadanos la aportación de datos y transmitiéndoles la percepción de que la Administración utiliza y contrasta la información de que ya dispone.

También la materialización del pago inicial de la pensión y la notificación de la resolución al mismo tiempo, han facilitado la simplificación de trámites burocráticos e influido directamente en la consecución de un procedimiento más ágil. Se cambia de un sistema mensual de primeros pagos a un sistema de emisión diaria que sitúa en el domicilio del interesado un cheque por el importe del primer pago añadiendo agilidad y comodidad al proceso. Con ello se produce la ruptura con la complejidad tradicional de la Administración para ejecutar sus propios actos.

La definición de un nuevo procedimiento de gestión de prestaciones económicas (PROGESPRESS) ha servido como instrumento para cambiar la cadena de producción de los expedientes, los sistemas de trabajo, los mecanismos de control y la estructura decisional de las personas que intervienen en la tramitación. Para mejor comprender la utilidad del mismo resulta ilustrativo hacer una pequeña referencia al anterior procedimiento cuyo esquema se incluye en la figura 1.

La multiplicidad de fases existentes indica que se trataba de un procedimiento excesivamente burocratizado en el que las posibilidades de mejora mediante la incorporación de modificaciones parciales resultaba absolutamente inoperante, ya que impedía agilizar la tramitación, exigía la incorporación y mantenimiento de masivos medios humanos, impedía la utilización de la red de centros periféricos, generaba resultados insatisfactorios y daba lugar a que hubiera múltiples decisiones, no siempre necesarias, adoptadas por personas distintas.
Ante esta situación se diseñó el PROGESPRESS cuyo esquema se incorpora en la figura 2. Este procedimiento tiene unos objetivos muy nítidos:

- Mejorar la información a los usuarios.

- Agilizar los trámites de resolución de las pensiones y prestaciones del sistema.

- Dotar de simplicidad la gestión de los expedientes.

- Eliminar el soporte papel que supone una ruptura con la tradición de construir un expediente, de firmar, sellar, registrar, etc., los diferentes documentos.

La experiencia obtenida en estos años es muy positiva y confirma las expectativas inicialmente depositadas en el mismo, mejorando de forma definitiva los flujos de información existentes entre las unidades de gestión y apoyo. Sus características principales son las siguientes:

- Concentra en una persona todas las responsabilidades relacionadas con los trámites que conlleva la apertura y análisis de la documentación.

- Supone la designación de una persona responsable de las decisiones de impulso y resolución de las solicitudes.

- Clarifica el procedimiento evitando los retrocesos en la gestión del expediente y suprime múltiples trámites innecesarios y repetitivos.

- Se soporta en aplicaciones informáticas que mantienen permanentemente comunicados todos los centros CAISS con la Dirección provincial, lo que permite una optimización de la red de centros mediante la oportuna reordenación de la gestión provincial.

- Permite un nivel de respuesta más ágil, de mayor calidad y precisión, a la vez que se posibilita la disminución de las personas dedicadas a la gestión de pensiones, liberándolas para la realización de nuevas actividades.

- Acaba con las comunicaciones interdepartamentales que se producían en número de millón y medio al año.

Hoy en día, la gestión de las más de 400.000 pensiones solicitadas al año son resueltas en base a este procedimiento sin que sea necesario trasladar documentos, solicitudes, notas internas, etc., desde una a otra unidad, pues las comunicaciones se efectúan por impulsos electrónicos entre puestos de trabajo informatizados pudiendo afirmar que realmente se trata de una Gestión sin papel.

Una reflexión retrospectiva sobre cuáles han podido ser las causas del éxito logrado, las fundamentaría básicamente, en las siguientes: 


\section{Figura 1}

\section{Procedimiento de gestión de las prestaciones económicas. Análisis del procedimiento anterior}

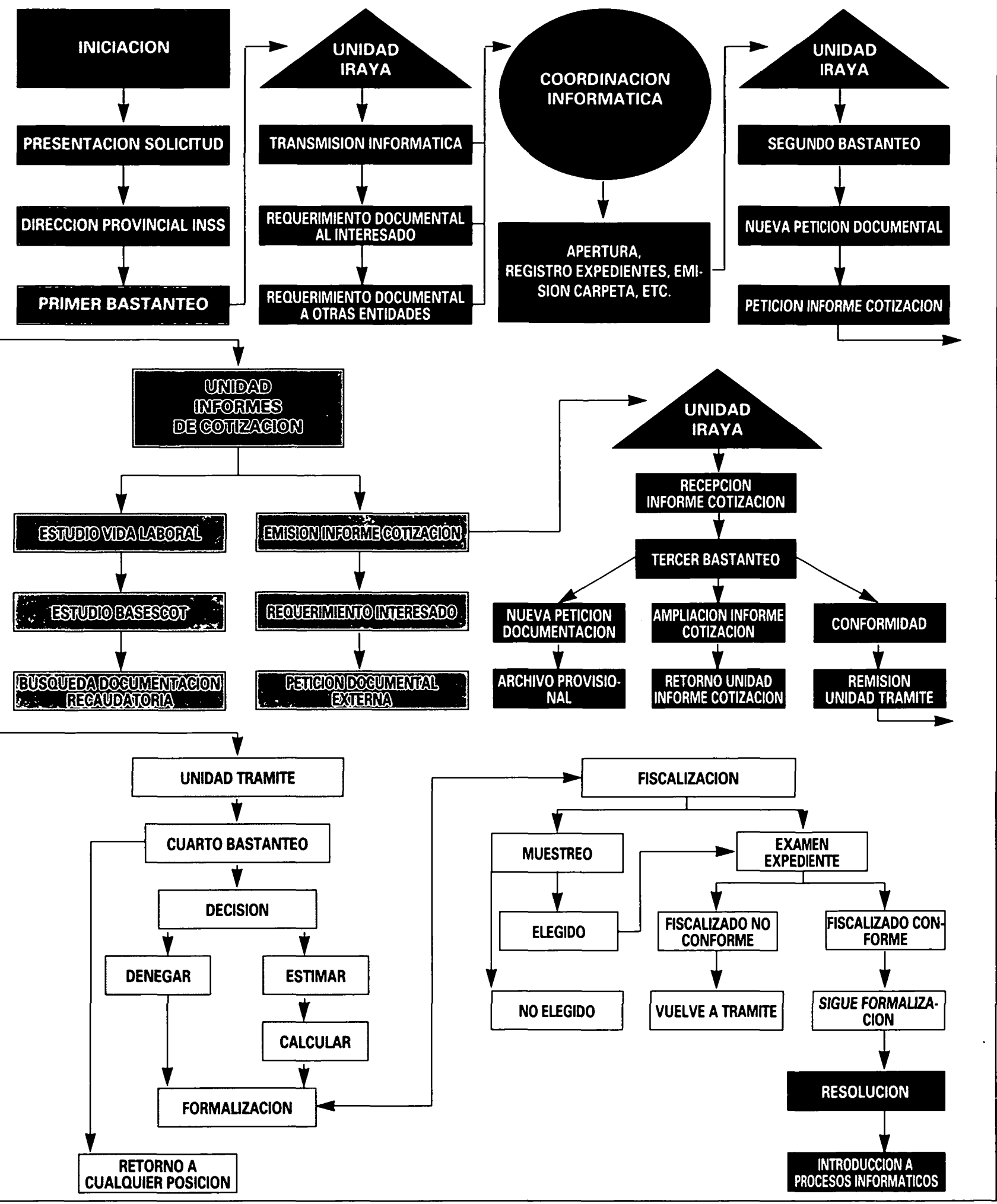




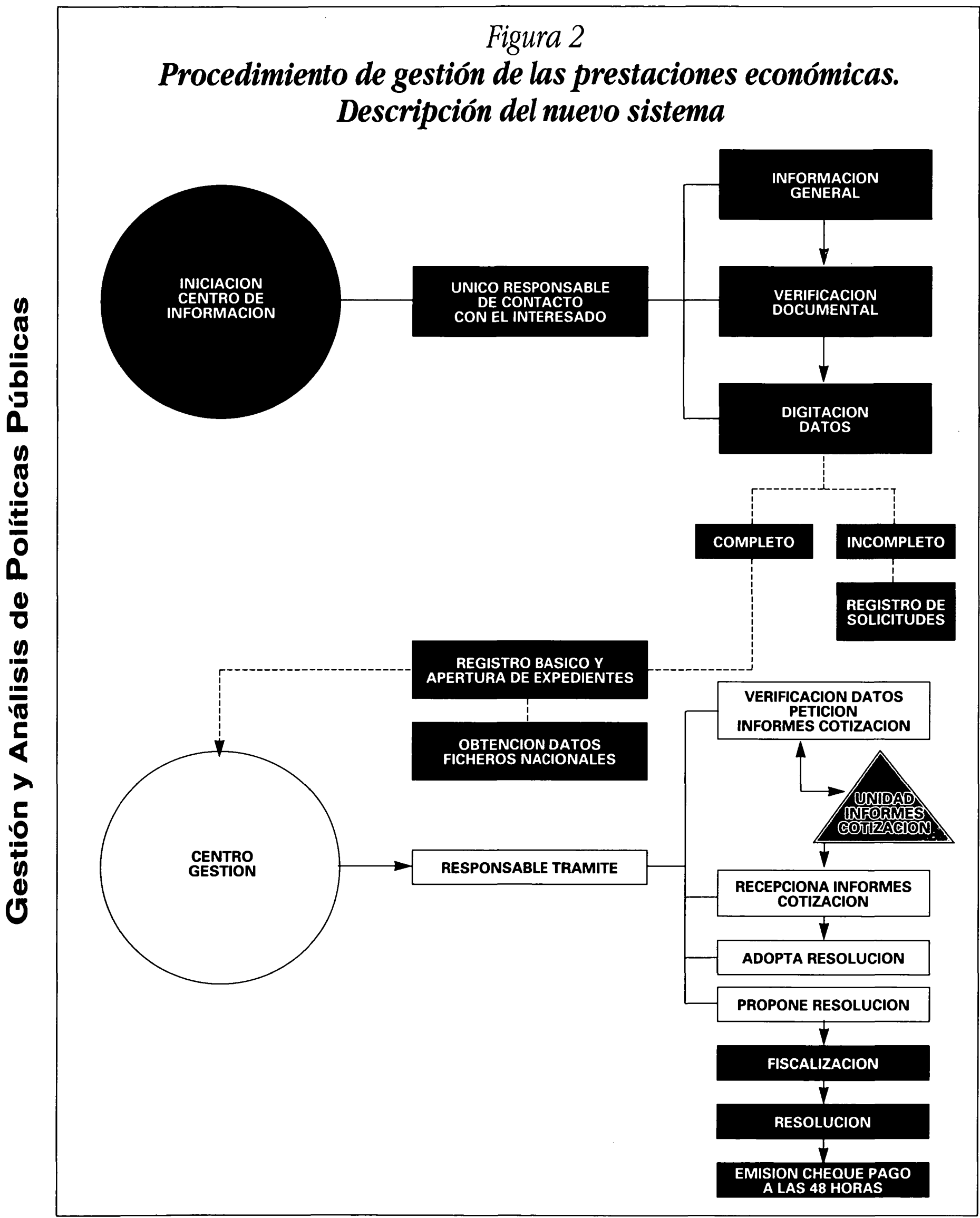


- Se produce una agrupación de las operaciones y actividades incluidas en cada fase del procedimiento de forma más lógica y coherente.

- iviantiente la capacidad de decisión humanáa en el ámbito de la aplicación de las disposiciones y la adopción de criterios jurídicos y económicos.

- Dota de seguridad a los empleados públicos que participan en las distintas fases del proceso, ya que desde el momento que se procede a incorporar la solicitud de prestación o pensión en la base de datos, todos y cada uno de los responsables pueden acceder a la información y conocer en qué situación se encuentra la tramitación del expediente y cuál es la causa por la que se encuentra en dicha fase.

- Atribuye a la aplicación informática las tareas de mero cálculo y control interno para evitar resultados no lógicos, suprimiendo las tareas administrativas manuales, repetitivas y lentas.

De este modo, la gestión del INSS ha ido incorporando paulatinamente, elementos de racionalidad de diversa indole que facilitan el mantenimiento de un alto grado de estabilidad gestora y de capacidad para predecir con anticipación las demandas de prestaciones y de pensiones.

\section{Las personas}

El nuevo modelo de organización, constituido esencialmente por los centros CAISS configurados como centros abiertos al ciudadano, donde se desarrollan las políticas de atención, información y apoyo a la gestión y las Direcciones provinciales como centros de producción cerrados al ciudadano que tramitan y reconocen las pensiones solicitadas, ha exigido la puesta en funcionamiento de políticas de recursos humanos que pudieran transmitir a los empleados públicos cuales son los objetivos que deben orientar a una Administración moderna cuyos rasgos distintivos deben ser: la eficacia, la eficiencia, la calidad, la transparencia, la accesibilidad y el compromiso con las demandas sociales de los ciudadanos.

De este modo, el desarrollo de las políticas de Atención e Información (en adelante $\mathrm{A}+\mathrm{I}$ ) se han constituido en la segunda palanca utilizada por la Institución para llevar a cabo el cambio gestor. Los distintos programas ejecutados han perseguido un doble objetivo: delimitar la oferta global de servicios de los CAISS y dotar a los empleados públicos de los medios para poder satisfacer las demandas de los ciudadanos.
La oferta de servicios de estos centros constituye la expresión más concreta y visible de cómo la organización considera que la actividad burocrática está al servicin de los clientes y dónde se puede percibir que el solicitante tiene más de cliente que de administrado. En este sentido, se inserta el actual contenido competencial de los CAISS constituido por:

- La emisión de información individualizada sobre las prestaciones del sistema.

- La atención personalizada a los usuarios y, en su caso, la colaboración en la cumplimentación de solicitudes y requisitos previstos.

- La recepción e incorporación al PROGESPRESS de las solicitudes de prestaciones presentadas.

- Atención, control y gestión de las peticiones, modificaciones e incidencias de todo tipo que afecten a los pensionistas del sistema.

De esta manera, los CAISS se configuran como el centro natural de los pensionistas y los demandantes de nuevas prestaciones y han hecho posible una mejor utilización de la red mediante la reasignación de los recursos humanos existentes en cada provincia.

De todos son conocidas las rigideces que en la Administración Pública existen para la movilidad de empleados de unos centros a otros, sobre todo cuando están situados en municipios distintos y, en este sentido, el INSS no es una excepción. Sin embargo, en los últimos años se ha logrado incorporar empleados públicos en la red de centros, a pesar de que en términos globales no ha habido un incremento de plantilla, sino una reducción de efectivos de al menos el 12 por 100 .

En cualquier caso, la creación de nuevos centros y la aplicación del PROGESPRESS han llevado a explorar nuevas vías en la gestión de las personas y la más importante de todas es, sin duda, la implantación de políticas de formación institucional.

Los programas de formación desarrollados en los últimos años están vinculados a las necesidades de la Institución y no sólo han servido como vehículo para la transmisión de conocimientos necesarios para el desempeño de las tareas inherentes al puesto de trabajo, sino que han permitido transmitir los objetivos que la organización entendía que deben incorporarse a la cultura de la misma. También la formación se ha transformado en una herramienta de motivación que los directivos utilizan en sus relaciones con sus colaboradores y empleados; así como de impulso de nuevas iniciativas surgidas 
en los niveles medios y básicos de la estructura organizacional.

Los procesos de formación adoptan diversas fórmulas a nivel central, zona básica y provincia y sus temáticas hacen referencia a: modernización de la Administración Pública, informática, gestión CAISS, conocimientos jurídicos, información y comunicación, gerencia pública, etcétera

En los últimos tres años se ha impartido una media de mil cursos al año, en los que el 74 por 100 se ha llevado a cabo con medios propios y el 26 por 100 restante con medios ajenos y el volumen de alumnos que, con carácter anual, ha recibido algún curso se incluye en la figura 3. La progresión a lo largo de los años ha sido constante habiendo participado en los tres últimos años un número de alumnos superior a la plantilla real de empleados de la Institución.

\section{Los recursos}

En una organización tan amplia y compleja como el INSS, los recursos y medios empleados para la consecución de sus fines son muy numerosos, si bien la especial importancia de algunos les coloca en una situación rele- vante frente a los demás ya que han tenido una influencia decisiva en el proceso de cambio implantado. Entre ellos cabe citar: los medios informáticos, los sistemas de información y la aplicación del sistema A+I en los centros de contacto.

El crecimiento de los medios informáticos del INSS durante los últimos años ha sido muy importante, ya que cada una de las 52 Direcciones Provinciales dispone de ordenadores de propósito general con aplicaciones específicas en las que se sustentan el PROGESPRESS, herramientas informáticas para el control de las pensiones y conexión con los bancos de datos del sistema de la Seguridad Social (Afiliación, Pensionistas del sistema y de Pensiones Públicas). Además, el parque de ordenadores personales se encuentra situado en más de 3.000 unidades, empleándose principalmente en aquellas actividades de apoyo a la gestión cuyas características aconsejan su utilización.

También se han incorporado las tecnologías basadas en la lectura de documentos mediante láser para su posterior almacenamiento en disco óptico. Actualmente son treinta y cuatro las provincias que disponen de esta tecnología comúnmente denominada sistema $M E G A D O C$, que permite la conservación de la documentación en discos ópticos

Figura 3

\section{Cuadro comparativo de asistentes. Planes de formación INSS 1987-1993}

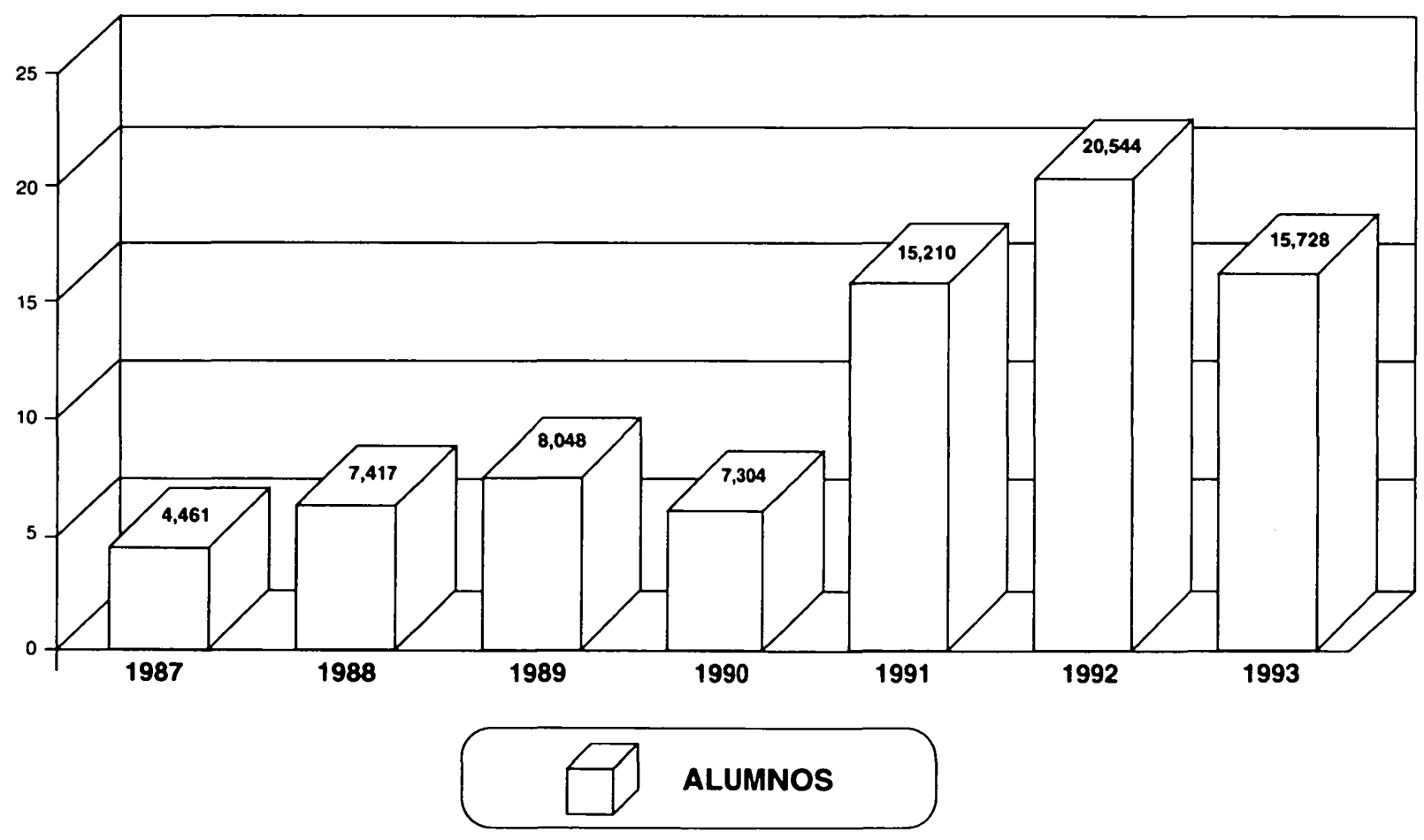


y su recuperación en cualquier momento, lo cual tiene claras ventajas para una organización como el INSS donde, a pesar de la drástica reducción de papel que supuso la simplificación y la incorporación de un procedimiento que permite gestionar los expedientes mediante impulsos electrónicos, aun dispone y recibe gran cantidad de documentos que debe clasificar, ordenar, comprobar, custodiar y conservar durante al menos cinco años. Pues bien, el sistema MEGADOC realiza todas esas funciones repetitivas que no generan casi valor añadido con un mínimo de participación de empleados públicos, hace más ágil la recuperación de información y reduce las necesidades de espacio físico dedicado a la conservación de documentos.

La creación de sistemas de información sobre las actividades relevantes de la organización es otra de las herramientas más desarrolladas en el INSS. Al disponer de una gestión descentralizada en las Direcciones provinciales, la incorporación de mecanismos de información sobre la actividad desplegada en cada una de ellas se convierte en un requisito imprescindible.
Por ello, y de forma paulatina, se han puesto en funcionamiento varios sistemas de información sobre: gestión de las prestaciones que se reconocen, gestión presupuestaria, gestión de ia atención directa y personaiizada en los centros, gestión de las pensiones ya reconocidas y control de los requisitos para su mantenimiento. Este grupo de sistemas han constituido una base estadística, cuyas series anuales, en algún caso, están formadas por varios años. Esta información ha facilitado la implantación de la dirección por objetivos (DPO), en las principales actividades.

De este modo, la Institución puede fijar los objetivos de gestión que desea obtener para el conjunto del Estado y para cada una de las Direcciones provinciales en el presente año y cuya determinación se lleva a cabo en función de diferentes indicadores. La utilización de estas técnicas ha resultado muy positiva para la organización ya que facilita la toma de decisiones, permite homogeneizar los resultados obtenidos en cada provincia y constituye una herramienta para los directivos de línea de

\section{Figura 4 \\ Sistema de atención e información}

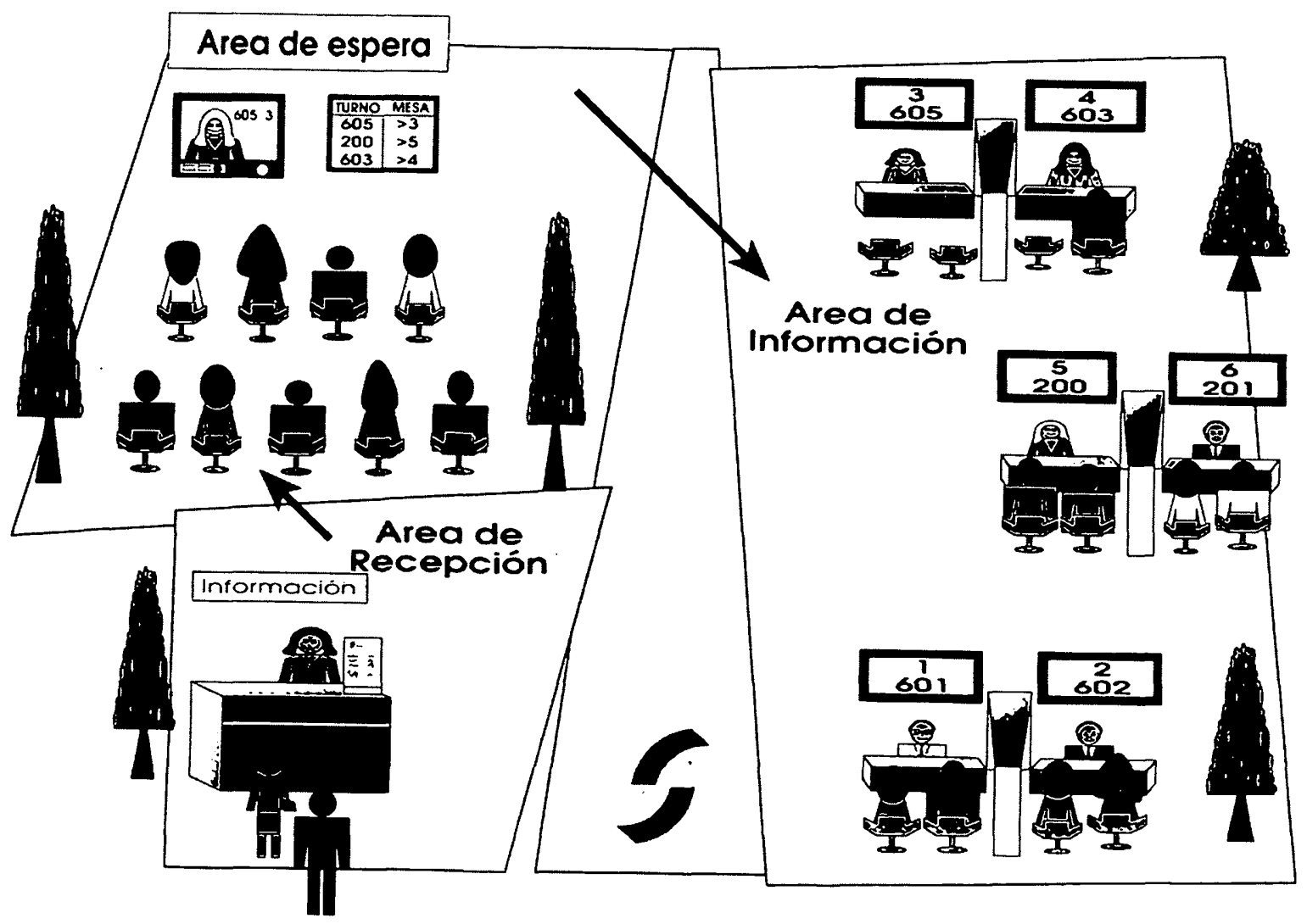


importancia considerable, a la hora de valorar los resultados y los rendimientos de las personas que forman parte de los grupos y equipos a su cargo.

Otro factor de importancia decisiva en el sistema de A+I implantado, lo constituye el diseño del entorno físico en el que se facilitan los servicios a los usuarios. Los centros incorporan varias áreas de servicio, según se puede ver en la figura 4, donde el usuario encuentra un entorno agradable y una gestión que percibe como rápida y eficaz. Estas áreas son:

- Área de recepción/entrada, situada a la entrada del patio de operaciones, donde el usuario expone sus demandas y recibe la primera información y desde donde le remiten a la zona de espera.

- Área de espera, en la que el cliente permanece sentado recibiendo informaciones complementarias a través de medios audiovisuales.

- Área de atención, donde es atendido por un empleado especializado en un entorno personalizado y confidencial.

El sistema mantiene un soporte informático de carácter estadístico de los usuarios atendidos y proporciona la información sobre la atención prestada a los mismos. Toda la información se puede obtener por prestación, día, hora, puesto de trabajo y permite, entre otras, conocer cuál es el número de clientes atendidos y no atendidos; los usuarios que han tenido que esperar; los clientes atendidos por puesto de trabajo, etc.

Actualmente, son más de 50 los CAISS que tienen en funcionamiento un sistema de estas características que facilita y dirige los flujos de clientes de forma más racional y cómoda hacia los puntos de atención.

Los primeros datos provisionales indican que el volumen de clientes atendidos se sitúa en torno a las 4.000 personas por centro/mes; el tiempo medio de espera oscila entre los tres y diez minutos; y el trabajo de atención entre los dos y catorce minutos.

Este sistema aporta múltiples beneficios a los usuarios, a los empleados públicos y a la Institución destacando como los más importantes los siguientes:

\section{a) Para el cliente}

- Sensación de recibir un servicio más personal, directo y confidencial.

- Sentirse bien informado.

\section{Figura 5}

\section{Expedientes de jubilación gestionados}

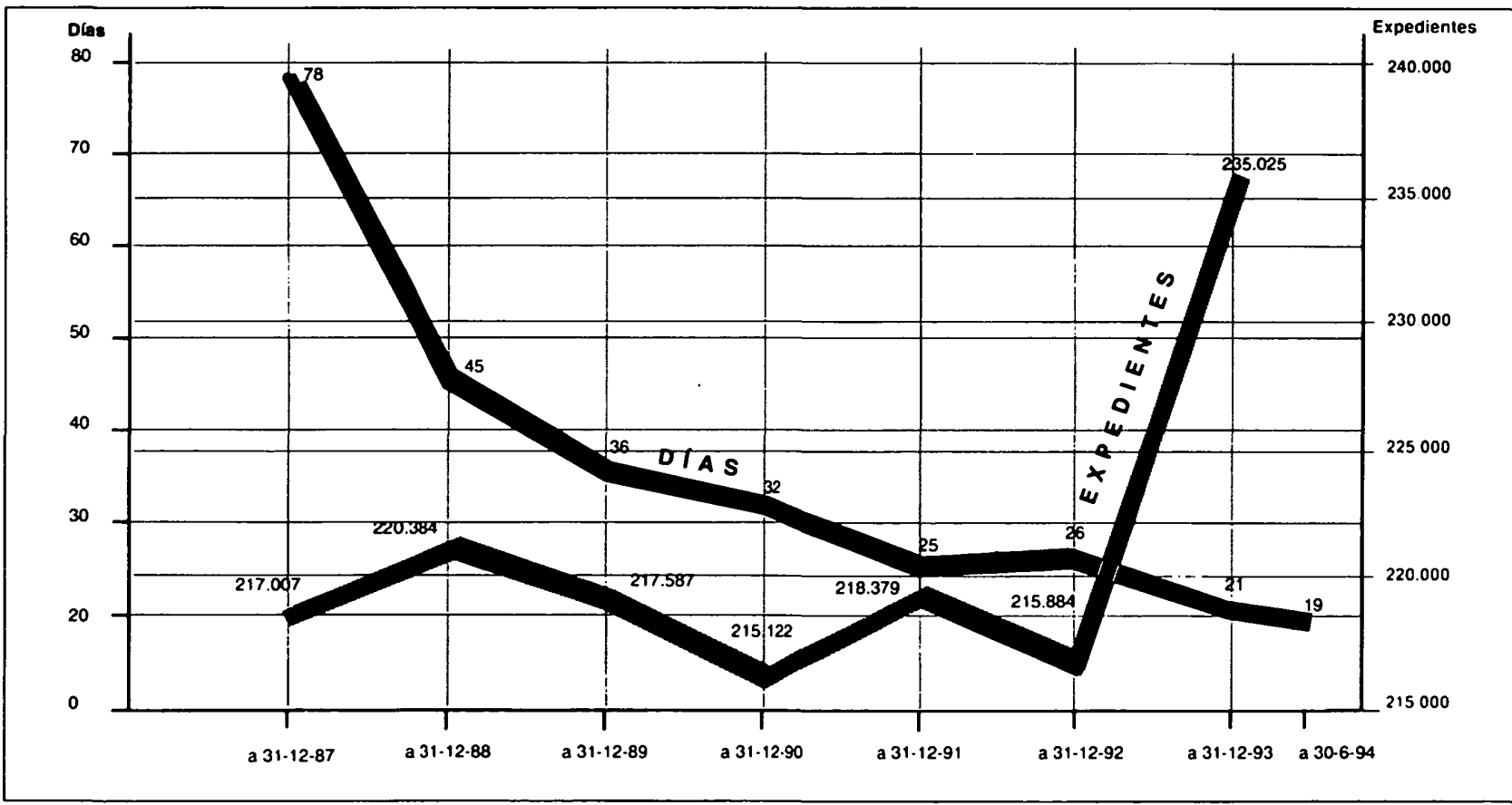

AÑOS $1987 \cdot 1994$ 
- Imagen de local ordenado y confortable.

- Tiempos de atención y respuesta más cortos.

- Áreas de espera adecuadas.

- Desaparece la sensación de estar siempre en la cola errónea.

- Disminución del stress.

b) Para los empleados públicos

- Reparto equitativo de la carga de trabajo.

- Elimina aglomeraciones en los puntos de servicio personalizado.

- Desaparece la sensación de agobio.

- Distribución programada de ausencias, control de presencia, pausas, turnos, etc.

- Reducción significativa del stress.

c) Para la Institución

- Mejora significativa de la imagen.

- Reorientación del servicio al usuario mediante el envío de mensajes, publicidad dinámica, campañas informativas, etc.
- Agilización de los tiempos de espera.

- Delimitación de objetivos específicos para la ejecución de los servicios.

- Racionalización en ìa distribución de ios recursos humanos.

- Imagen homogénea de los CAISS.

\section{Los resultados obtenidos}

La implantación de las políticas que se citaban al principio de este trabajo es indudable que ha producido unos resultados muy positivos en la gestión de las pensiones, tal como muestran los gráficos que sobre la evolución de la pensiones de jubilación, viudedad y orfandad se incluyen en las figuras 5 y 6 .

El reconocimiento de las pensiones se ha agilizado de forma muy importante, ya que su duración en 1987 era de setenta y ocho dias en jubilación y sesenta en supervivencia (agrupa viudedad y orfandad); mientras que en 1993 se empleaban diecinueve y once días, respectivamente. Con lo cual, se ha conseguido alcanzar el objetivo

\section{Figura 6 \\ Expedientes de supervivencia gestionados}

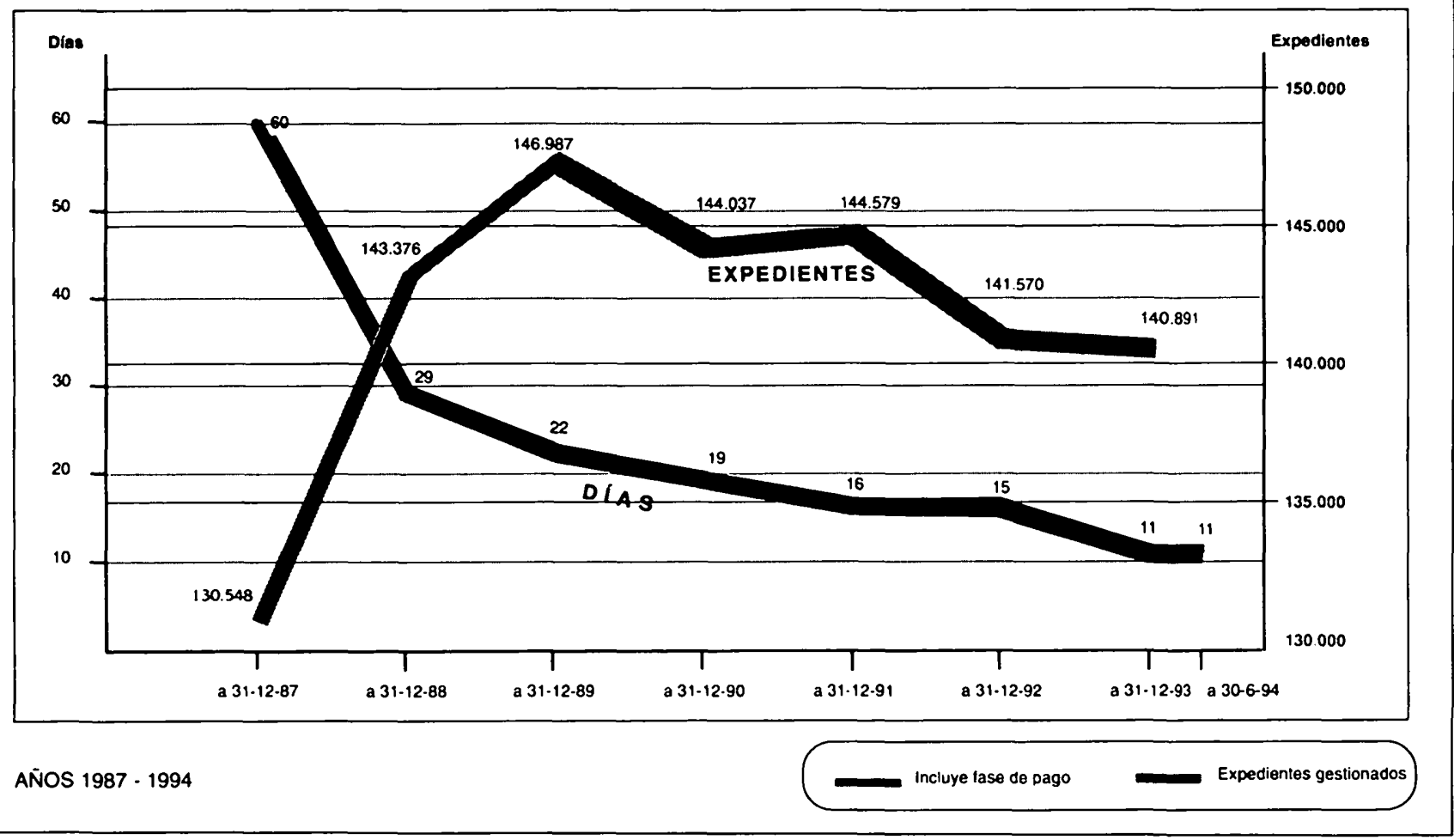


finalista consistente en que no se produzca una interrupción entre la extinción del salario y el cobro de la pensión reconocida.

También el examen de estos gráficos muestra que la tendencia a disminuir los tiempos de gestión se suaviza en los últimos años, lo cual es indicativo de que la gestión de las pensiones tiende a estabilizarse en torno a los veinte días con lo que se garantiza el cumplimiento del objetivo finalista (la interrupción de rentas se produce cuando se superan los treinta días), en una actividad que se puede calificar no solo de ágil, sino de muy eficaz y altamente eficiente.

Igualmente, el intenso proceso de formación llevado a cabo entre los empleados públicos ha contribuido en gran medida a la obtención de dichos resultados, así como a la configuración de una cultura organizacional en la que la prestación de servicios al cliente no resulta un concepto ajeno, si bien su aceptación y comprensión han resultado relativamente más sencillas en una Institución gestora de servicios como el INSS.

Por último, las inversiones realizadas en instalaciones físicas, nuevas tecnologías y en la informatización masiva de la red periférica que, a finales de 1993, alcanzaba al 92 por 100 de los centros, han coadyuvado a la transformación de la gestión y, en definitiva, a las relaciones que mantienen los empleados públicos y los ciudadanos demandantes de servicios.

\section{La opinión del usuario}

Después de lo dicho hasta aquí, parece lógico pensar que la organización ha realizado un esfuerzo considerable para convertirse en una Administración moderna, receptiva, accesible y eficaz; sin embargo... ¿qué piensan los destinatarios de los servicios, ¿basta qué punto se sienten satisfechos?

En un primer momento, el análisis de las sugerencias y quejas llevado a cabo en los últimos años ponía de manifiesto la existencia de ciertas debilidades importantes en la gestión de los servicios, tales como: la existencia de un período de tiempo excesivo en la gestión y pago de las pensiones; la ausencia casi total de información sobre los derechos de los usuarios, que los situaba en una auténtica posición de indefensión; la existencia de procesos complejos con exigencia de muchos documentos cuya exigencia legal era, cuando menos, dudosa; el trato y cortesía dispensado a los usuarios presentaba múltiples deficiencias lo cual se veía agravado por la formación de colas que provocaba en los usuarios disgusto y una valoración negativa del servicio recibido.

La necesidad de comprobar si las medidas adoptadas habian permitido superar las debilidades citadas, y si las necesidades de los usuarios respecto de la forma de prestar el servicio se habían cumplido, es lo que aconsejó la puesta en marcha de un sistema de evaluación de la calidad de los servicios prestados por medio del cual

\begin{tabular}{|c|c|c|c|}
\hline $\begin{array}{c}\text { Figura } 7 \\
\text { Clientes satisfech }\end{array}$ & & & \\
\hline Indicador & $\begin{array}{c}1991 \\
- \\
\text { Porcentaje }\end{array}$ & $\begin{array}{c}1992 \\
- \\
\text { Porcentaje } \\
\end{array}$ & $\begin{array}{c}1993 \\
- \\
\text { Porcentaje }\end{array}$ \\
\hline Tiempo empleado en la atención y tramitación........ & 94,0 & 92,1 & 93,9 \\
\hline 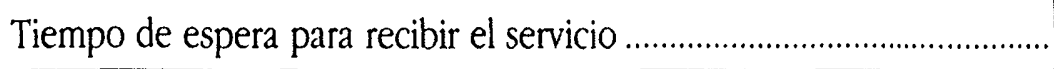 & 75,5 & 77,4 & 79,4 \\
\hline 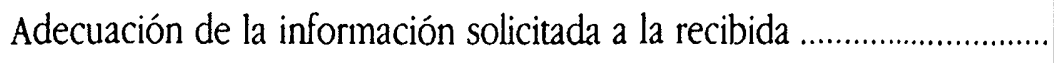 & 72,3 & 76,1 & 74,7 \\
\hline Simplificación documental ....................... & 93,1 & 93,5 & 94,5 \\
\hline Comprensión de la notificación/ facilidad para ejercer los derechos ....... & 89,1 & 91,6 & 90,2 \\
\hline Calidad de la atención prestada . & 78,9 & 83,1 & 82,4 \\
\hline Valoración global del procedimiento gestor ........ & 91,9 & 92,9 & 92,4 \\
\hline
\end{tabular}


fuera posible determinar el nivel de satisfacción e insatisfacción de los clientes.

Para ello se diseñó un cuestionario dirigido a los usuarios que adquieren la condición de nuevos pensionistas, con la finalidad de conocer su opinión sobre el nivel de calidad de los servicios utilizados. El mismo es remitido junto con la resolución de la pensión al domicilio del ciudadano que, una vez cumplimentado, lo devuelve a la Institución en sobre de franqueo gratuito.

La información obtenida y analizada en los tres últimos años se incluye en la figura 7 . En este período de tiempo se han recibido más de 125.000 cuestionarios, siendo 1993 el año en el que se han recibido mayor número (47.280 cuestionarios).

La favorable respuesta obtenida de los ciudadanos que utilizan el principal servicio del INSS sirve a los responsables de la organización de estímulo para profundizar en la línea iniciada, ya que es el mejor camino para conseguir una Institución eficaz, eficiente en el cumplimiento de sus obligaciones y legitimada por la opinión de los ciudadanos a quienes se presta servicios.

\section{Conclusiones}

Para terminar, creo que es conveniente resaltar que la potenciación en la cultura del INSS de la figura del ciudadano-cliente es lo que ha facilitado la regeneración de las actividades de $\mathrm{A}+\mathrm{I}$; impulsoras de la prestación de servi- cios y ha permitido a los empleados públicos que las desarrollan, percibir la importancia que la Institución da al trabajo que ellos realizan todos los días.

La incorporación de tecnologías modernas constituye un soporte de primerísima importancia en la racionalización de la gestión, así como en el mantenimiento de altos niveles de agilidad y eficacia, y ello a pesar de la reducción de efectivos humanos y del incremento sustancial de la gestión encomendada a la Entidad.

También la formación permanente de las personas que constituyen la organización y la incorporación de herramientas gestoras de la información, ha robustecido la capacidad de decisión y el liderazgo del personal directivo y de muchos empleados públicos que han encontrado en la formación sistemática un medio de autorrealización personal y de mejora profesional.

Sin embargo, esto no significa que el proceso esté cerrado, todo lo contrario, una organización tan grande como el INSS siempre tiene problemas por resolver, lo importante es que de alguna manera la estrategia de futuro se encuentra trazada y se dirige, básicamente, a la consolidación de los logros obtenidos, al incremento de los niveles de calidad de la gestión y de satisfacción de los usuarios, a la mejora de los sistemas de control en el acceso y en el mantenimiento de las prestaciones, la racionalización del gasto, el incremento de la cooperación con el resto de la Administración Pública, la formación permanente, la incorporación de nuevas tecnologías a los procesos de gestión y la búsqueda de la mejora continua de la calidad en los procesos de gestión.

* Secretario general del INSS. 


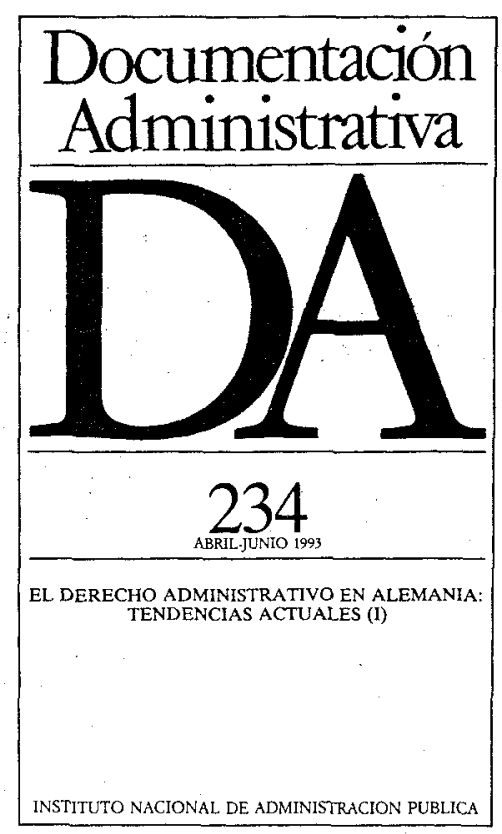

Publicación trimestral del Instituto Nacional de Administración Pública, especializada en Derecho Administrativo y Organización y Gestión de las Administraciones Públicas.

Aparecida en 1958, la característica más destacada de su actual etapa es la dedicáción monográfica de cada número a una cuestión específica, que se analiza desde diferentes puntos de vista por acreditadas firmas de España y otros países.

\section{CONSEJO DE REDACCION}

Presidente: Alejandro Nieto García. Vocales: Manuel Aragón Reyes, Enrique Argullol Murgadas, Miguel Beltrán Villalva, Andrés de Blas Guerrero, José Manuel Castells Arteche, Rafael Gómez-Ferrer Morant, Luis Morell Ocaña, José Ramón Parada Vázquez, Juan Alfonso Santamaría Pastor, Gumersindo Trujillo Fernández.

\section{Director}

\section{Luciano Parejo Alfonso}

Secretario

Jesús Prieto de Pedro

\section{Edita:}

Instituto Nacional de Administración Pública

ISSN: 0012-4494

NIPO: 329-94-001-5

Depósito legal: M 581-1958

\section{Distribución y suscripciones:}

Instituto Nacional de Administración Pública Subdirección General de Publicaciones José Marañón, 12. 28010 MADRID Teléfono: (91) 4461700 Fax: (91) 4450839

\section{Precios:}

Suscripción anual (4 números) ........ 4.000 pts

Número sencillo 